\title{
Effects of Aerobic Exercise on Body Composition at Different Phases of the Menstrual Cycle
}

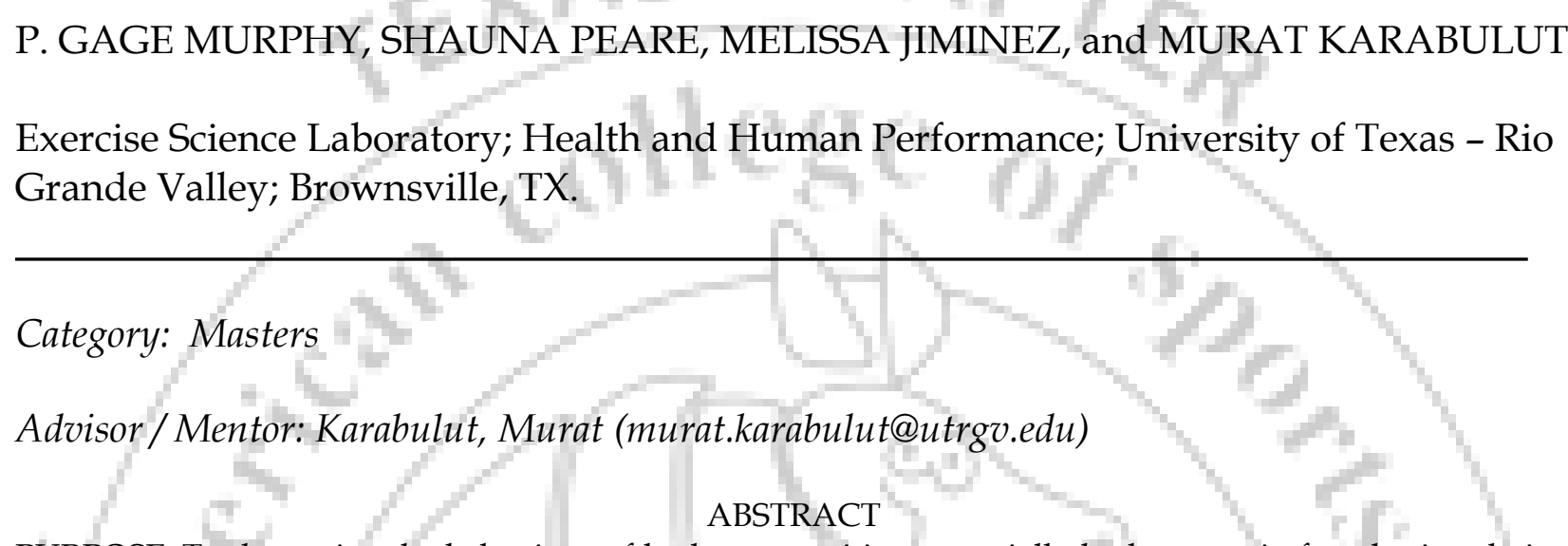

PURPOSE: To determine the behaviour of body composition, especially body water in females in relation to their menstrual cycle and in response to aerobic exercise. METHODS: Eight females (age $=20.4 \pm 1.6$ yrs) performed four sessions of aerobic activity at $70 \%$ of their calculated $\mathrm{VO}_{2}$ max. $\mathrm{VO}_{2}$ max was determined by utilizing the Bruce Protocol. The speed for each subject's aerobic activity was then calculated using the ACSM metabolic running equation. The four sessions of aerobic activity were identical in format and performed on days 1, 7, 14, and 21 of each subject's menstrual cycle. Sessions began with measurements of body weight, body fat, extracellular fluid (ECF), intracellular fluid (ICF), and fatfree mass (FFM) via single-frequency and multiple-frequency bio-electrical impedance analysis (BIA) prior to aerobic activity. Subjects then completed 30 minutes of aerobic activity at the calculated speed to yield an intensity of $70 \% \mathrm{VO}_{2}$ max. Following the aerobic activity, all measures taken pre-exercise were measured again at three separate time points post-exercise; post-0min, post- $15 \mathrm{~min}$, and post-30min. This combined for a total of 4 separate time points at which measurements were taken; pre, post-0min, post$15 \mathrm{~min}$, and post-30 min. RESULTS: Significance was seen for a condition difference in body mass $(\mathrm{p}<0.04)$ and for a time difference in ECF $(p<0.01)$ and body fat $(p<0.01)$. Body mass significantly decreased from Day 1 to Day 7 and ECF volume was significantly changed from time point pre to post-0min and post$15 \mathrm{~min}$ on all days. No condition main effects were seen for body water measurements, but a trend for condition*time interaction was seen in ICF difference pre-post $(p=0.09)$. CONCLUSIONS: Body water responses to aerobic exercise varied across the days of the menstrual cycle, which caused fluctuations in body weight, ECF, and ICF. The percent changes in ICF values from pre to post-30min were increased by $3.2 \%$ on day 14 and decreased by $5.6 \%$ on day 7 . ICF behaviour on different days suggests that the menstrual cycle may have impacts on performance. Further investigation is required to back up such claims. Future studies should investigate the effects of different duration and/or intensity of aerobic exercises on body composition along with blood pressure, heart rate, and RPE.

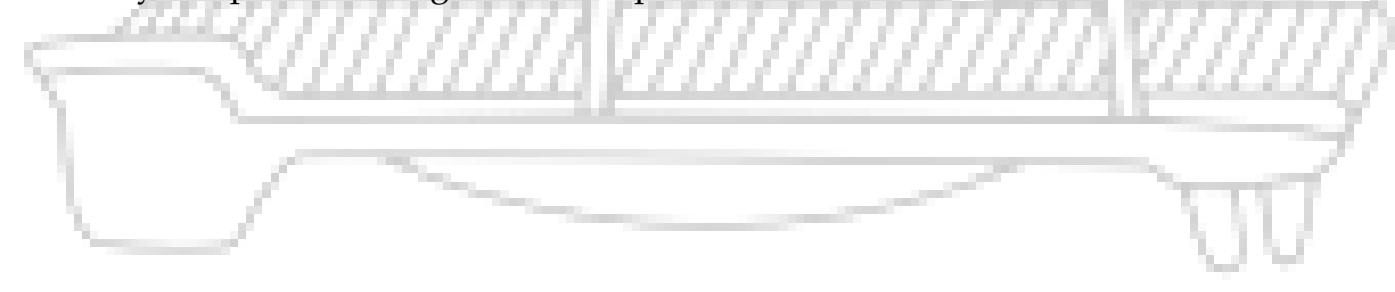

